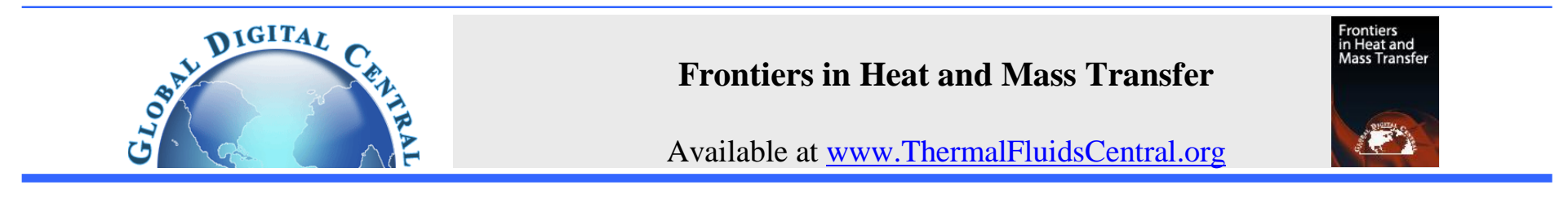

\title{
CFD-BASED STUDY ON HEAT TRANSFER ENHANCEMENT BEHIND A PROJECTION IN A MINIATURIZED FLOW CHANNEL BY PULSATING FLOW
}

\author{
Wakana Hiratsuka ${ }^{a}$, Takashi Fukue ${ }^{\text {b,* }}$, Hidemi Shirakawac, Katsuyuki Nakayama ${ }^{\text {d }}$, Yasushi Koito ${ }^{\mathrm{e}}$ \\ ${ }^{a}$ Former student, Iwate University, Iwate 020-8551, Japan \\ ${ }^{b}$ Kanazawa Institute of Technology, Ishikawa 921-8501, Japan \\ ${ }^{c}$ National Institute of Technology, Toyama College, Toyama 939-8630, Japan \\ d Toyama 939-0398, Japan \\ e Kumamoto University, Kumamoto 860-8555, Japan
}

\begin{abstract}
This paper describes a possibility of heat transfer enhancement in a mm-scale flow channel by using a combination of some projections and pulsating flow. The objective of this research is to develop a novel heat exchanger for miniaturized productions such as high-density packaging electronic equipment by applying pulsating flow to enhance heat transfer while inhibiting an increase of pressure drop. In order to evaluate the possibility of applying pulsating flow to miniature water channels, a three-dimensional flow and heat transfer analysis was performed. Heat transfer performance of a combination of pulsating water flow and a projection was investigated. The mechanism of heat transfer enhancement by the proposed combination was also investigated. The proposed combination enhances heat transfer in the channel remarkably. Heat transfer performance of pulsating flow around the projection was approximately $80 \%$ higher than that of steady flow in the channel without the projection. This was caused by the generation of counter flow behind the projection during the deceleration period. The counter flow ejected heated water near the projection and heat transfer enhancement is achieved.
\end{abstract}

Keywords: Heat Exchanger, Heat Transfer Efficiency, Pulsating Flow, Projection, Thermal Design of Electronic Equipment.

\section{INTRODUCTION}

Due to an increase of demanding of energy saving to build a sustainable society and further improvement of performance and functions of products such as electrical vehicles, air conditioning systems and electronic equipment, an innovation of heat transfer enhancement technology by forced convection is strongly demanded (e.g., Jaluria et al., 2020). Thermal management of electronic equipment is one of applications of forced convection. Forced convection by air or water is widely used for removing heat from electronic equipment (e.g., Panchal et al., 2017). In recent years, both downsizing and design improvement of electronic equipment have been required in order to respond to various requests by users. For instance, portable electronic devices, such as laptop personal computers, tablet computers, smartphones, and portable POS (point of sale) terminals, have become smaller and thinner whereas their functions have become more complex. As a result, the mounting density of electrical devices has significantly increased whereas the applicable cooling method has become limited because there is little space to mount cooling devices (Ditri et al., 2015; Fukue et al., 2010; Yang et al., 2015). In this regard, a novel heat exchanger that features high heat exchange performance and small dimensions is desired against the increase of heat dissipation in miniature devices in the near future. Several types of devices of heat transfer enhancement (e.g., Aliaga et al., 1994; Bi et al., 2013; Colleoni et al., 2010; Reddy et al., 2015, etc.) have been widely investigated. Especially, several types of ribs are widely used for forced convection heat transfer enhancement. Zhao and Tian
(2019) have investigated the heat transfer enhancement of a chip heat sink by the combination of the ribs. Al-Turaihi et al., (2019) have evaluated the effects of semi-circle ribs on heat transfer enhancement. of These devices have shown high potential as the heat transfer enhancement methods for forced convection. However, in miniaturized heat exchangers, the flow passages of working fluid is narrow. The flow condition is laminar flow and the effect of viscosity is dominant. Therefore, an influence of pressure drop becomes more distinct. The above heat transfer enhancement devices also increase pressure drop. These devices generally enhance heat transfer by generating turbulence. However, in the narrow flow passages, the effect of viscosity becomes higher and the generation of turbulence is difficult. Therefore, the devices work as not the heat transfer enhancement device but obstructions of the flow. Hence, a novel technique that can enhance heat transfer in narrow flow passages while inhibiting the increase of pressure drop should be developed.

Several studies (e.g., Saitoh et al. (2010); Inukai et al. (2005); Kikuchi et al. (1995); Shiibara et al. (2016)) have investigated special characteristics of pulsating flow from the viewpoint of heat transfer enhancement. A pulsating impinging jet was also investigated and the effects of heat transfer enhancement have been reported (e.g., Fukue et al., 2016; Hofmann et al., 2007; Liewkongsataporn et al., 2008). Souma et al. (2010) have reported pressure drop reduction of turbulent flow in the pipe by flow pulsation. As one of heat transport devices, several researches on pulsating heat pipes have been reported (e.g., Goshayeshi et al., 2016). It is possible that the application of pulsating flow to nextgeneration heat exchangers for miniaturized thermal equipment would be 
effective.

However, in order to apply pulsating flow to the miniaturized heat exchangers, an effectiveness of pulsating flow in the actual flow passages in the heat exchangers should be investigated. In our previous research (Fukue et al., 2014), we experimentally investigated the heat transfer performance of pulsating air flow around a cylindrical block that simulated electronic components. It was found that the heat transfer performance around the cylindrical brock can be increased in the case of $\mathrm{cm}$-scale pulsating air flow. As the next step, the effects of working fluid and the scale of flow passage on the heat transfer performance by pulsating flow should be investigated. It is beneficial to clarify whether or not pulsating flow is available for mm-scale flow channels that can be observed in the miniaturized heat exchangers and water flows as coolant. However, the experimental investigation of pulsating water flow in mmscale channels is generally difficult due to the control of the generation of the flow pulsation.

Against these backgrounds, in this study, we focused on heat transfer in a water-cooling channel by pulsating flow from the viewpoint of improvement of heat exchange performance of miniaturized heat exchangers. As a preliminary investigation, a 3-dimensional CFD (Computational Fluid Dynamics) analysis of pulsating water flow in a $\mathrm{mm}$-scale channel was carried out. In order to enhance heat transfer in the miniaturized channel while inhibiting pressure drop increase, we evaluated the possibility of heat transfer enhancement by pulsating flow in a simple square duct and a duct with a square projection, which can be used as heat transfer enhancement devices in a narrow duct. In order to investigate the mechanism of heat transfer enhancement by pulsating flow, the difference in flow pattern between steady water flow and pulsating flow was also examined. We clarified the possibility of applying pulsating flow to the miniaturized heat exchangers as a nextgeneration heat transfer enhancement method.

\section{ANALYTICAL MODEL}

Figure 1 shows the analytical models investigated in this study. The dimensions of the analytical model simulated those of a flow channel in a miniature heat exchanger for cooling device of high heat flux electronic chips. The working fluid was water. In this study, in order to investigate the possibility of heat transfer enhancement by pulsating flow in a mmscale flow channel with small flow rate, transient laminar flow and heat transfer analysis in the low Reynolds number condition was performed. A square flow path having flow channel width and height of $5 \mathrm{~mm}$ was investigated. Two models were prepared: (a) was a simple square channel model to examine the possibility of heat transfer enhancement by pulsating flow in a simple channel (hereinafter called "Non-projection model") and (b) was a channel with a projection (hereinafter called "Projection model"). The dimensions of the projection were $2.5 \mathrm{~mm}$ in height, $5.0 \mathrm{~mm}$ in width, and $2.5 \mathrm{~mm}$ in length. A constant heat flux was applied to the heating area set $30 \mathrm{~mm}$ behind the inlet boundary, as shown in Fig. 1. This simulated heat dissipation from an electrical chip mounted on the bottom of the heat exchanger. The length of the heating area was $70 \mathrm{~mm}$ including the projection in the case of Projection model. Here, the surface of the projection did not have any heat flux. This simulated less expensive materials, such as resin, which has low thermal conductivity. This was intended to construct water flow channel in an actual heat exchanger by using low-cost materials. Then, heat transfer performance in the heating area was compared. Here, an additional flow channel of optimum length was prepared upstream and downstream of the heating area, respectively, in order to decrease the effects of the inlet and outlet boundary conditions on the flow and heat transfer on the heating area. Mesh number was approximately 180,000. As the CFD code, OpenFOAM $^{\circledR}$, an open-source CFD toolbox, was used. As the solver, buoyantBoussinesqPimpleFoam, one of the OpenFOAM solver that transient thermal-fluid flow can calculate, was used. The governing equations were the equation of continuity, the Navier-Stokes equation and the energy equation (The Open CAE Society of Japan, 2016):

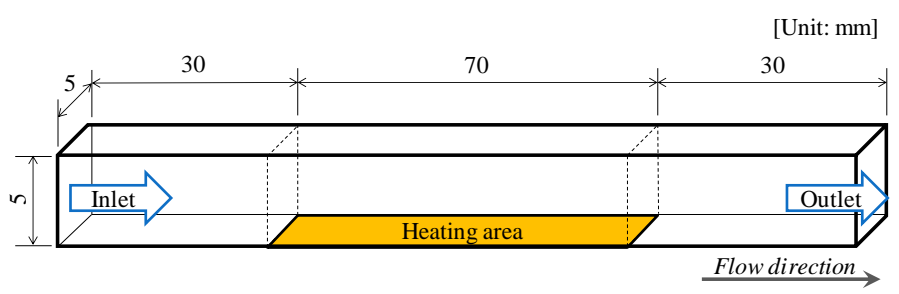

(a) Non-projection model

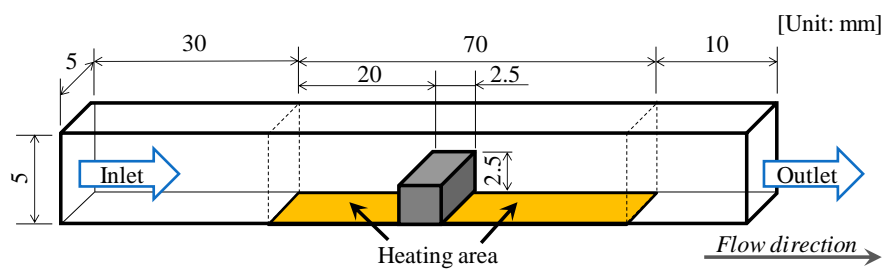

(b) Projection model

Fig. 1 Analytical models of mini channel investigated in this study.

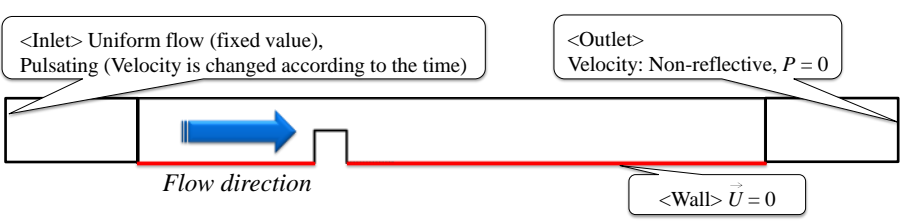

(a) Flow boundary

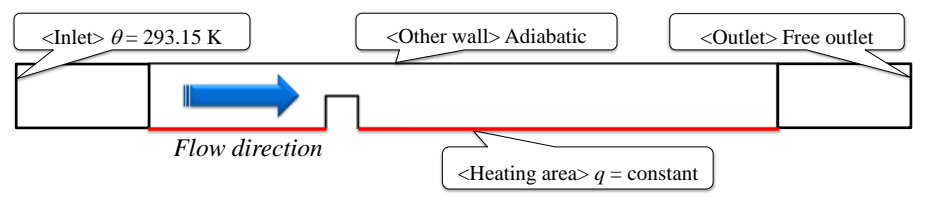

(b) Temperature boundary

Fig. 2 Boundary conditions of each analytical model.

(a) Equation of Continuity

$$
\nabla \cdot \vec{U}=0
$$

(b) Navier-Stokes Equation

$$
\frac{\partial \vec{u}}{\partial t}+\nabla \cdot(\vec{u} \vec{u})=-\nabla P+\nabla \cdot\left[v\left\{\nabla \vec{u}+(\nabla \vec{u})^{T}\right\}\right]
$$

(c) Energy Equation

$$
\frac{\partial \theta}{\partial t}+\nabla \cdot(\theta \vec{u})=\nabla \cdot(\alpha \nabla \theta)
$$

where $\vec{U}[\mathrm{~m} / \mathrm{s}]$ is velocity vector, $t[\mathrm{~s}]$ is time, $p\left[\mathrm{~m}^{2} / \mathrm{s}^{2}\right]$ is static pressure divided by reference density, $v\left[\mathrm{~m}^{2} / \mathrm{s}\right]$ is kinematic viscosity, $\theta[\mathrm{K}]$ is temperature and $\alpha\left[\mathrm{m}^{2} / \mathrm{s}\right]$ is thermal diffusivity.

Figure 2 shows the boundary conditions of the proposed analytical model. An inlet boundary was set at the upstream side of the analytical model. The outlet was the Non-reflective boundary condition (Kasuga, 
2015; Nozaki, 2019) and other walls were the no-slip wall condition. Regarding the boundary conditions for heat transfer, the flow inlet was the constant temperature boundary. The flow outlet was the free outlet boundary. The heating surface, which is the bottom surface of the flow passage and corresponds to the cooling surface of the water-cooling device, was the constant heat flux boundary, and the top and side walls were the thermal insulation boundary.

\section{ANALYTICAL CONDITIONS}

Table 1 shows the analytical conditions. Figure 3 shows the time variation of the flow rate of pulsating flow. The following time-averaged Reynolds number was $25,50,100$, and 150 . Here, the subscript " $t$ " means an instantaneous value and the top bar means a time-averaged value.

$$
\overline{R e_{\mathrm{d}}}=\frac{1}{t} \int_{\mathrm{t}} \frac{u_{\mathrm{c}, \mathrm{t}} d_{\mathrm{c}}}{v} d t
$$

where $u_{\mathrm{c}}[\mathrm{m} / \mathrm{s}]$ is the bulk mean flow velocity at the inlet, $d_{\mathrm{c}}[\mathrm{m}]$ is the hydraulic diameter of the flow channel and $t[\mathrm{~s}]$ is time. In this report, $d_{\mathrm{c}}$ was defined as the hydraulic diameter at the inlet boundary of the models. Pulsating frequency was set to $1 \mathrm{~Hz}$ and $2 \mathrm{~Hz}$. The waveform of the flow rate was a trapezoidal wave, the maximum flow rate was reached in 0.1 second from the start of acceleration, and the flow rate became zero in 0.1 second from the start of deceleration. The instantaneous maximum flow rates in the case of the pulsating flow ( $V_{1 \max }$ and $V_{2 \max }$ in Fig. 3) were higher than the maximum flow rate of the steady flow in order to match the time-averaged flow rate to that of the steady flow analysis. The definitions of transient supply flow change $V_{\mathrm{c}, \mathrm{t}}\left[\mathrm{m}^{3} / \mathrm{s}\right]$ during 1 pulsating period are as follows:

- Pulsation frequency $=1 \mathrm{~Hz}: 1$ Pulsation period $=1 \mathrm{sec}$.

$$
\begin{array}{ll}
V_{\mathrm{c}, \mathrm{t}}=10 V_{1 \max } t & (0 \mathrm{sec} . \leq t \leq 0.1 \mathrm{sec} .) \\
V_{\mathrm{c}, \mathrm{t}}=V_{1 \max } & (0.1 \mathrm{sec} .<t \leq 0.4 \mathrm{sec} .) \\
V_{\mathrm{c}, \mathrm{t}}=10 V_{\max }(0.5-t) & (0.4 \mathrm{sec} .<t \leq 0.5 \mathrm{sec} .) \\
V_{\mathrm{c}, \mathrm{t}}=0 & (0.5 \mathrm{sec} .<t \leq 1.0 \mathrm{sec} .)
\end{array}
$$

- Pulsation frequency $=2 \mathrm{~Hz}: 1$ Pulsation period $=0.5 \mathrm{sec}$.

$$
\begin{array}{ll}
V_{\mathrm{c}, \mathrm{t}}=10 V_{2 \max } t & (0 \mathrm{sec} . \leq t \leq 0.1 \mathrm{sec} .) \\
V_{\mathrm{c}, \mathrm{t}}=V_{2 \max } & (0.1 \mathrm{sec} .<t \leq 0.15 \mathrm{sec} .) \\
V_{\mathrm{c}, \mathrm{t}}=10 V_{2 \max }(0.25-t) & (0.15 \mathrm{sec} .<t \leq 0.25 \mathrm{sec} .) \\
V_{\mathrm{c}, \mathrm{t}}=0 & (0.25 \mathrm{sec} .<t \leq 0.5 \mathrm{sec} .)
\end{array}
$$

\section{EVALUATION METHOD}

Heat transfer performance on the bottom of the channel was evaluated by using the following local Nusselt number $\left(N u_{x}\right)$ and the average Nusselt number $\left(\mathrm{N} u_{\mathrm{m}}\right)$.

$$
\begin{gathered}
N u_{x, \mathrm{t}}=\frac{h_{x, \mathrm{t}} d_{\mathrm{c}}}{\lambda} \\
h_{x, t}=\frac{q}{\theta_{x, t}-\theta_{\mathrm{in}}}
\end{gathered}
$$

Table 1 Analytical conditions.

\begin{tabular}{|l|l|}
\hline Working fluid & Water \\
\hline$\overline{R e_{\mathrm{d}}}$ & $25,50,100,150$ \\
\hline Heat flux $\left[\mathrm{W} / \mathrm{m}^{2}\right]$ & 4300 (Non-projection model) \\
& 4400 (Projection model) \\
\hline Pulsating frequency & Steady, $1 \mathrm{~Hz}, 2 \mathrm{~Hz}$ \\
\hline Mesh number & Approx. 180,000 \\
\hline
\end{tabular}

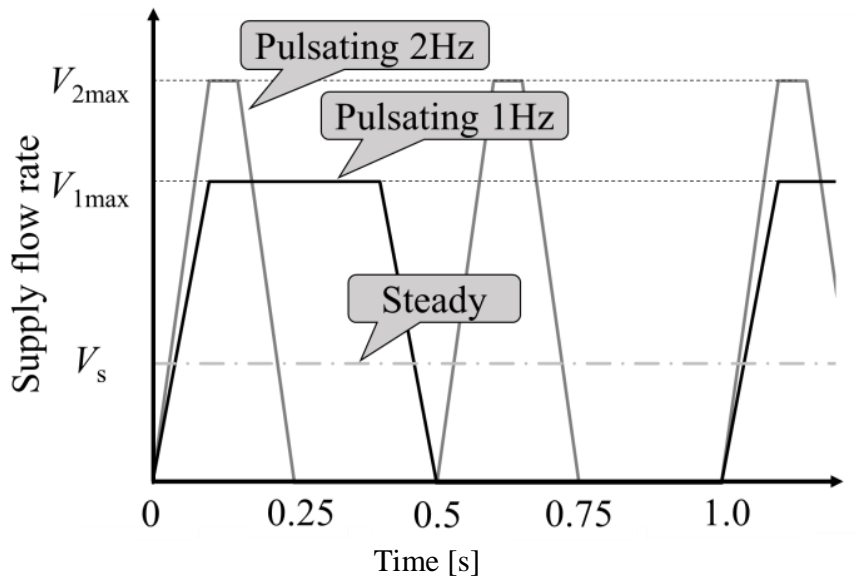

Fig. 3 Time variation of flow rate of pulsating flow.

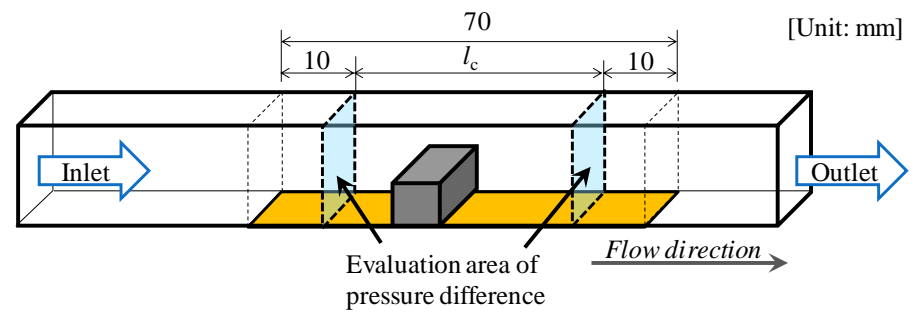

Fig. 4 Position of evaluation area of pressure drop.

$$
\overline{N u_{x}}=\frac{1}{t} \int_{t} N u_{x, \mathrm{t}} d t
$$

$$
\overline{N u_{\mathrm{m}}}=\frac{1}{A} \int_{A} \overline{N u_{x}} d A
$$

where $h_{x}\left[\mathrm{~W} /\left(\mathrm{m}^{2} \cdot \mathrm{K}\right)\right]$ is the local heat transfer coefficient, $\lambda[\mathrm{W} /(\mathrm{m} \cdot \mathrm{K})]$ is thermal conductivity of the working fluid, $\theta_{x}[\mathrm{~K}]$ is local temperature on the heating area, $\theta_{\mathrm{in}}[\mathrm{K}]$ is inlet fluid temperature, $q\left[\mathrm{~W} / \mathrm{m}^{2}\right]$ is heat flux and $A\left[\mathrm{~m}^{2}\right]$ is heating area. In this research, inlet temperature was used to define the heat transfer coefficient. In Eqs. (15) and (16), it was set as the average value for 1 second in a state where the flow was stable and the time variation of $N u_{x, t}$ of 1 second ( 1 process at $1 \mathrm{~Hz}, 2$ processes at $2 \mathrm{~Hz}$ ) did not change. The evaluation range of the average Nusselt number was set to $10 \mathrm{~mm}$ to $60 \mathrm{~mm}$ from the start of the heating area of the Projection model and the Non-projection model.

In addition, pressure drop in the channel was evaluated. The pressure difference between the upstream side and the downstream side of the channel as shown in Fig. 4 was evaluated. The level of the pressure drop was evaluated by using the following time-averaged friction coefficient $f_{\mathrm{c}}$ that a normalized friction loss in the flow passage by dynamical pressure. 


$$
\overline{f_{\mathrm{c}}}=\frac{1}{t} \int_{t} \frac{2 \Delta P_{\mathrm{c}, \mathrm{t}}}{\frac{d_{\mathrm{c}}}{l_{\mathrm{c}}} \rho u_{\mathrm{c}, \mathrm{t}}{ }^{2}} d t
$$

where $\Delta P_{\mathrm{c}}$ is the pressure drop in the channel and $\rho\left[\mathrm{kg} / \mathrm{m}^{3}\right]$ is water density. $l_{\mathrm{c}}[\mathrm{m}]$ is the distance between the evaluation points.

In addition, in order to evaluate net heat transfer performance by pulsating flow, the net heat transfer rate per temperature difference $E$ was defined as the following formula.

$$
E=\overline{h_{\mathrm{m}}} A
$$

Furthermore, the net pumping power $W$ was defined as the following formula.

$$
W=\overline{\Delta P_{\mathrm{c}}} \cdot \bar{V}_{\mathrm{c}}
$$

where $V_{\mathrm{c}}\left[\mathrm{m}^{3} / \mathrm{s}\right]$ is the time-averaged volume flow rate and $\overline{h_{\mathrm{m}}}\left[\mathrm{W} /\left(\mathrm{m}^{2} \cdot \mathrm{K}\right)\right]$ is the average heat transfer coefficient was defined as the following formula.

$$
\overline{h_{\mathrm{m}}}=\frac{\overline{N u_{\mathrm{m}}} \cdot \lambda}{d_{\mathrm{c}}}
$$

\section{ANALYTICAL RESULTS AND DISCUSSION}

\subsection{Time-Averaged Local Nusselt Number of Non-Projection Model}

We investigate the effect of pulsating flow on heat transfer in the case of Non-projection model. Figure 5 shows the relationship between timeaveraged local Nusselt number and the position of the evaluation point on the heating area. We can see that there is no difference in timeaveraged local Nusselt number between pulsating flow and steady flow. Of course, the transient temperature distribution on the heating surface is changed according to the generation of the pulsation. However, from Fig. 5 , we can say that time-averaged heat transfer performance of pulsating flow in the simple duct does not depend on the pulsating frequency. The net heat transfer performance depends on Reynolds number. In this case, because the pulsating frequency is slow, the hot fluid near the wall cannot be ejected effectively by the generation of the pulsating flow.

\subsection{Time-Averaged Local Nusselt Number of Projection Model}

Next, heat transfer performance of pulsating flow in the case of Projection model will be investigated. Figure 6 shows the results of timeaveraged local Nusselt number in the case of Projection model. Here, the gray broken lines denote the position of the projection. In the case of steady flow, an increase of heat transfer behind the projection can be confirmed regardless of the generation of pulsating flow. This is caused by the mixing of the fluid flow by the projection. Due to the existence of the projection, heat transfer behind the projection is enhanced.

On the other hand, in the case of pulsating flow, much higher heat transfer enhancement behind the projection is confirmed regardless of the time-averaged Reynolds number. In particular, the result for $2 \mathrm{~Hz}$ shows the highest local Nusselt number. The position of the highest local Nusselt number slightly changes according to the time-averaged Reynolds number. However, in the range of this study, the level of change of the position is small relative to the dimensions of the projection. In addition, in the case of $R e_{\mathrm{d}}=100$ and 150, local heat transfer

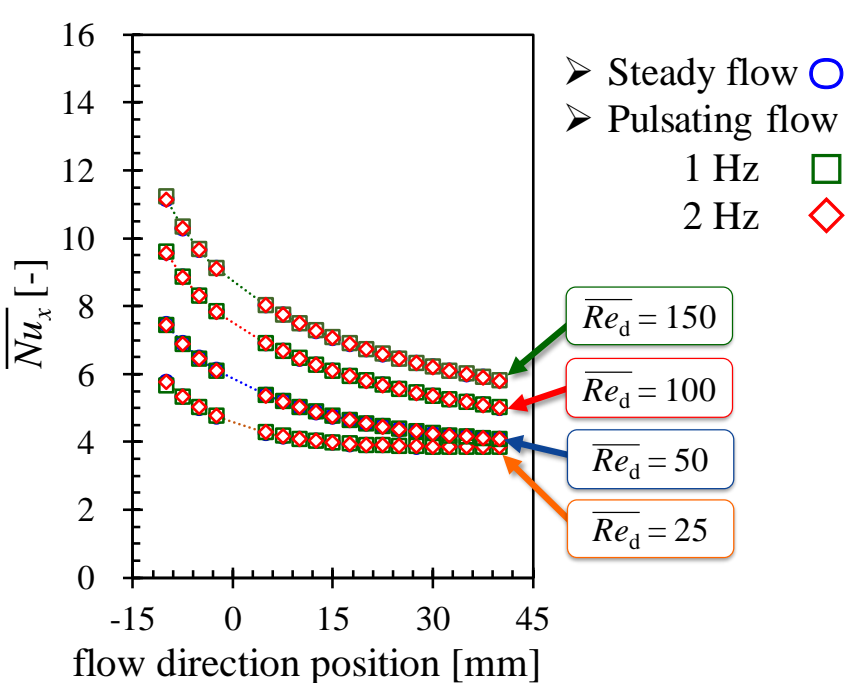

Fig. 5 Relationship between time-averaged local Nusselt number distribution and position in the case of Non-projection model.
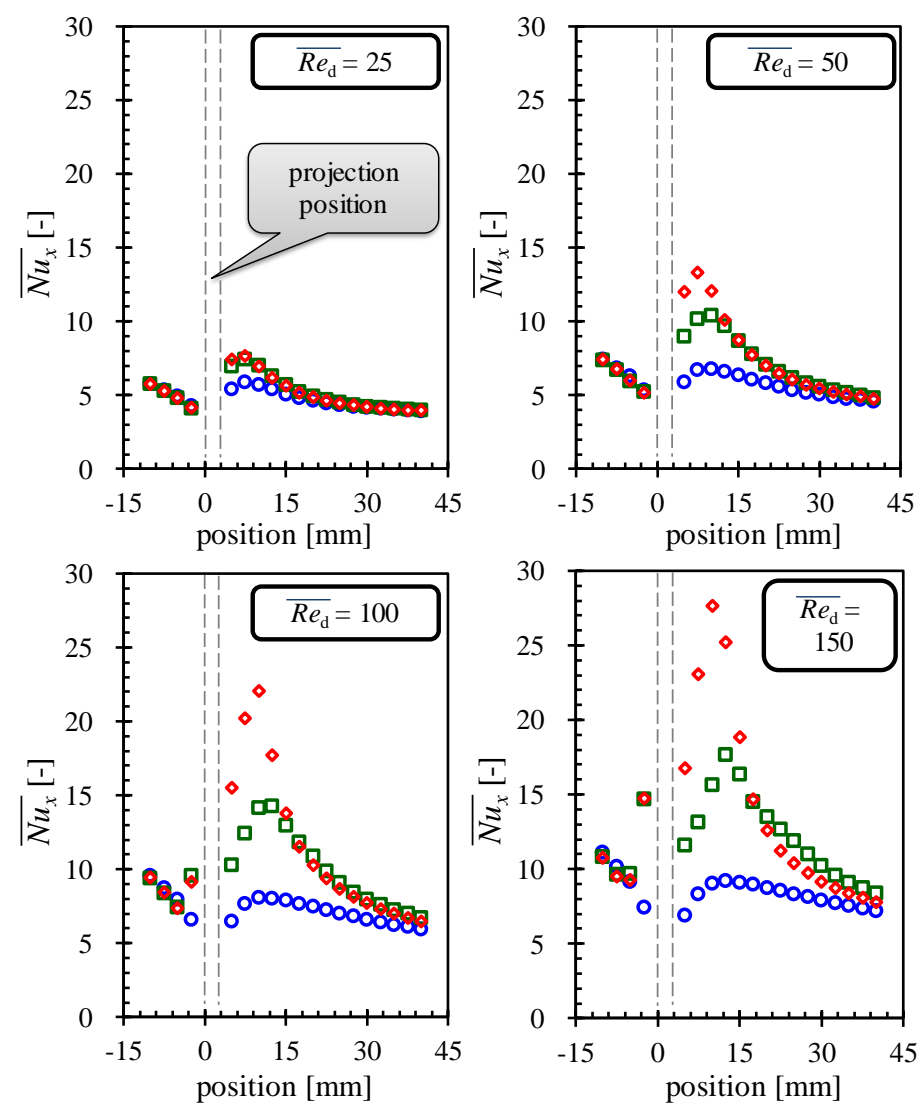

O Steady $\square 1 \mathrm{~Hz} \quad \diamond 2 \mathrm{~Hz}$

Fig. 6 Relationship between time-averaged local Nusselt number distribution and position in the Projection model.

performance increases in the area not only behind the projection but also upstream of the projection.

\subsection{Mechanism of Heat Transfer Enhancement by Pulsation}

In order to determine why pulsating flow enhances heat transfer around the projection, we will observe the flow pattern for one period of pulsating flow and the results of steady flow. Figure 7 shows the change of flow pattern around the projection. Figure 8 also shows the change of temperature distribution of working fluid around the projection. Here, 


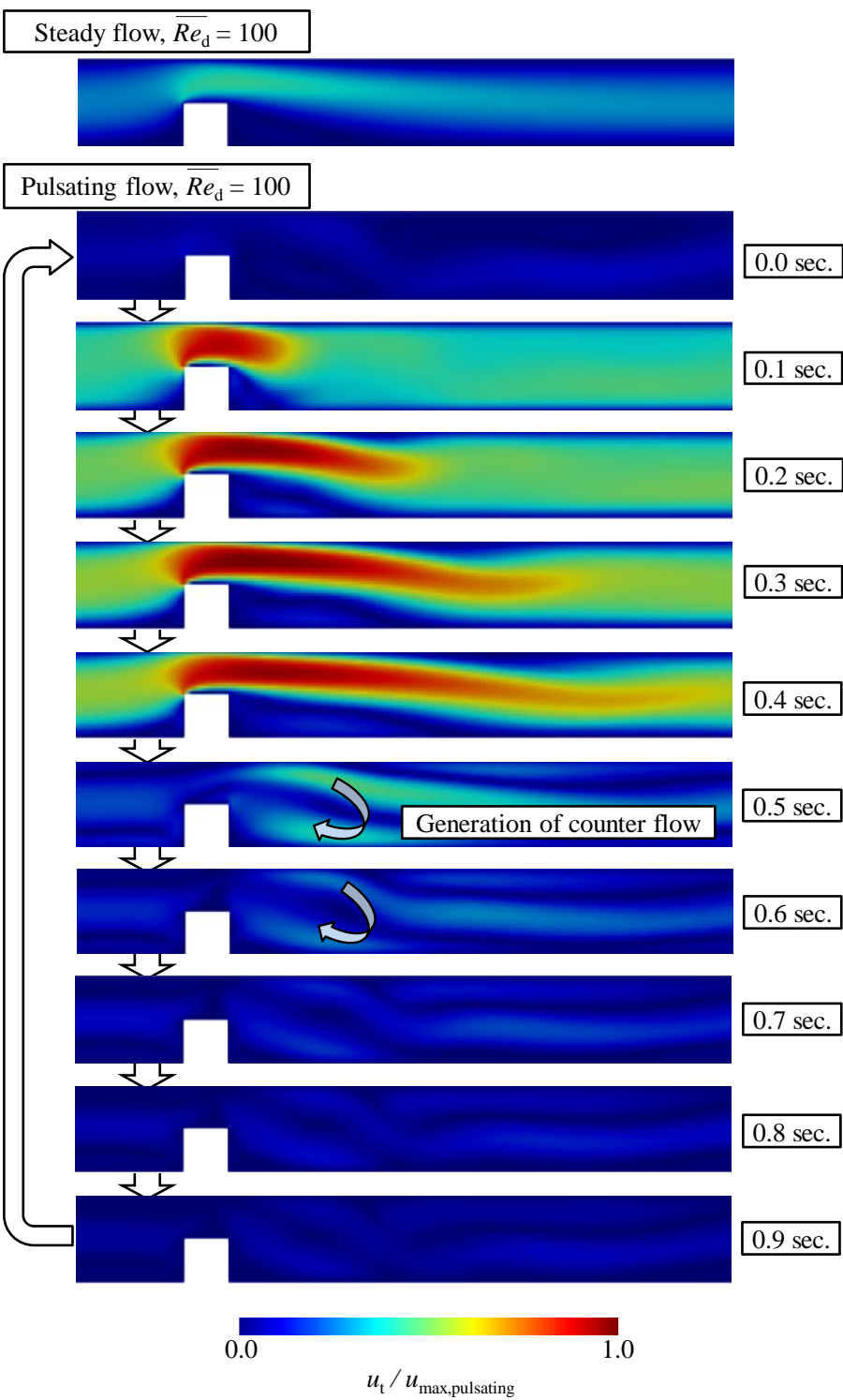

Fig. 7 Change of velocity distribution in the case of Projection model and $1 \mathrm{~Hz}$ pulsating frequency. The color of the velocity contour indicates the magnitude of the flow velocity. Here, the velocity was normalized by the maximum velocity magnitude in the case of $1 \mathrm{~Hz}$. Time-averaged Reynolds number is 100 .

about the contours in the pulsating flow, the results of the pulsating frequency in $1 \mathrm{~Hz}$ and the time-averaged Reynolds number in 100 was denoted.

In the case of steady flow, a flow separation behind the projection can be observed. In this area, a vortex is generated and rotates. This vortex mixes the flow behind the projection and causes higher heat transfer performance than the performance of Non-projection model. However, the flow between the projection and the top wall of the duct does not become complex and a laminar flow pattern can be observed. In addition, the area of flow separation is kept constant. The low Reynolds number condition is one of the reasons for maintaining the condition of the vortex behind the projection.

On the other hand, in the case of pulsating flow, the transient change of the flow pattern can be observed. During the acceleration period, we can observe that the flow separation behind the projection slightly develops with time. This also means that the vortex in the separation area is also developed. The vortex development mixes the flow and enhances heat transfer behind the projection. On the other hand, in the deceleration period, we can find a counter flow behind the projection in the case of

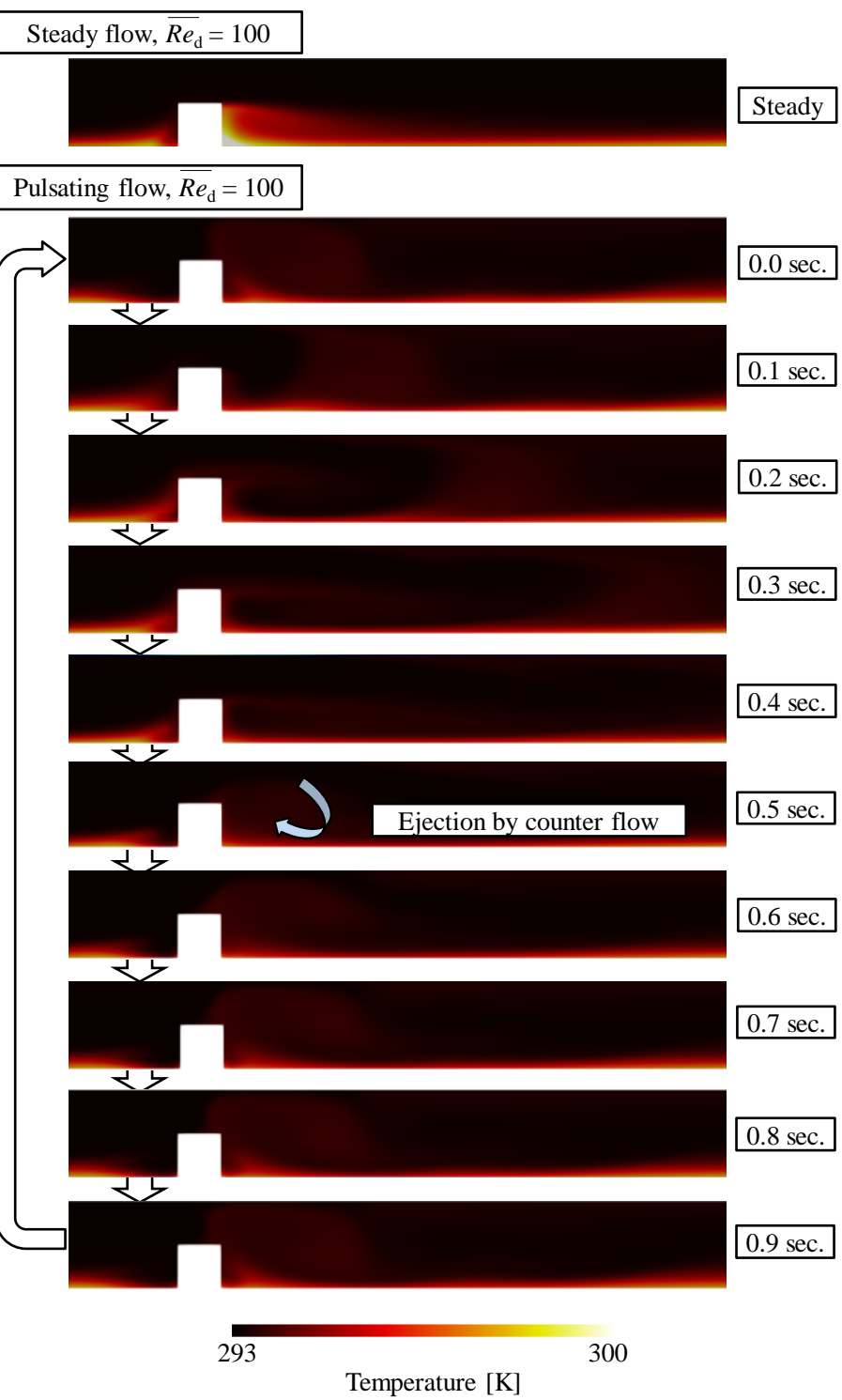

Fig. 8 Change of temperature distribution in the case of Projection model and $1 \mathrm{~Hz}$ pulsating frequency. Time-averaged Reynolds number is 100 .

pulsating flow. In addition, the vortex generated by the counter flow develops over the whole height direction of the flow passage. This flow pattern is different between pulsating flow and steady flow. In the deceleration period, the dynamical pressure of the main flow decreases due to the decrease of velocity. Thus, total pressure is not changed and static pressure increases. On the other hand, total pressure behind the projection becomes lower than that of the main flow because of flow separation. Therefore, when static pressure of the main flow increases with the decrease of flow velocity, a static pressure difference between the main flow and the flow separation area behind the projection is induced and flow can be generated by the occurrence of the sudden pressure difference in the deceleration period. This induces a counter flow and enhances heat transfer behind the projection.

We will also confirm the temperature distribution in the working fluid. In the case of the steady flow, higher temperature can be observed behind the projection. On the other hand, when the pulsating flow causes, the high temperature fluid near the rear wall of the projection is ejected from the separation area to the main flow pass by the counter flow. The ejected high temperature water is transported to the downstream by the main flow. This mechanism of the ejection of high temperature working fluid can supply cold working fluid to the downstream side of the 


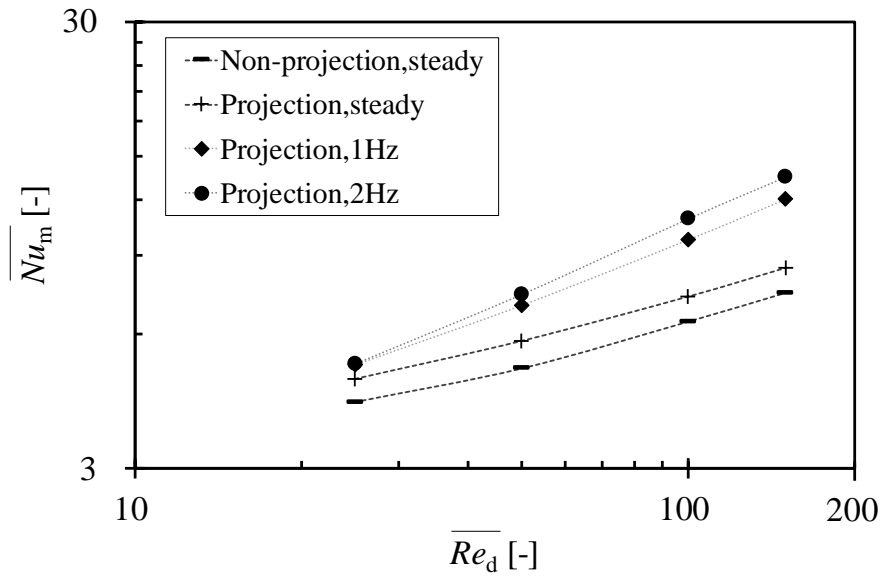

Fig. 9 Comparison of time-averaged average Nusselt numbers on the heating surface.

projection continuously and enhances heat transfer performance rear the projection.

\subsection{Average Heat Transfer Coefficient}

We will investigate average heat transfer coefficient in the channel heating area. Figure 9 shows the time-averaged average Nusselt numbers on the heating surface. About the steady flow analysis, the average Nusselt number of Projection model becomes higher than Non-projection model. This is caused by the generation of the complex flow by the projection. Furthermore, when pulsating flow is supplied, heat transfer enhancement by the projection becomes higher than the case of steady flow regardless of the Reynolds number. When pulsating frequency is 2 $\mathrm{Hz}$ and $R e_{\mathrm{d}}=150$, the time-averaged Nusselt number for the combination of projection and pulsating flow is $82 \%$ higher than that for the combination of steady flow and Non-projection model.

From these results, we conclude that the combination of pulsating flow and projection is effective for enhancing heat transfer in a narrow flow passage. In addition, the results obtained for $2 \mathrm{~Hz}$ pulsating frequency indicate higher heat transfer enhancement than the results for $1 \mathrm{~Hz}$. This is because the impingement number of the counter flow becomes higher as a result of the higher frequency and the level of heat transfer enhancement becomes higher.

\subsection{Net Heat Transfer Performance}

Finally, we evaluate an effectiveness of the pulsating flow as a heat transfer enhancement method from the viewpoint of net heat transfer performance including the pressure drop characteristic in the channel.

Figure 10 shows the relationship between the results of the friction coefficient of each analytical condition and the time-averaged Reynolds number. Here, in Fig. 10, the friction coefficient of fully-developed laminar flow in ducts $(=64 / R e)$ was also denoted. We can confirm that the friction drop in the channel is increased by the existence of the projection and the generation of the pulsating flow. About the effect of the projection on the pressure drop, the projection becomes an obstruction in the channel. Due to the existence of the projection, the main flow passage near the projection become narrower. This causes not only the heat transfer enhancement but also the pressure drop. Furthermore, when the pulsating flow is caused, the friction coefficient increases. This is caused by the sudden acceleration of the flow during the acceleration period of the pulsating flow. During the acceleration period, velocity gradient near the wall becomes higher and friction stress becomes higher. This causes the increase of the friction coefficient. This is a weak point of the application of pulsating flow to heat exchangers. Here, the analytical result of the friction coefficient of the steady flow in Non-projection model is higher than the friction coefficient of fully-

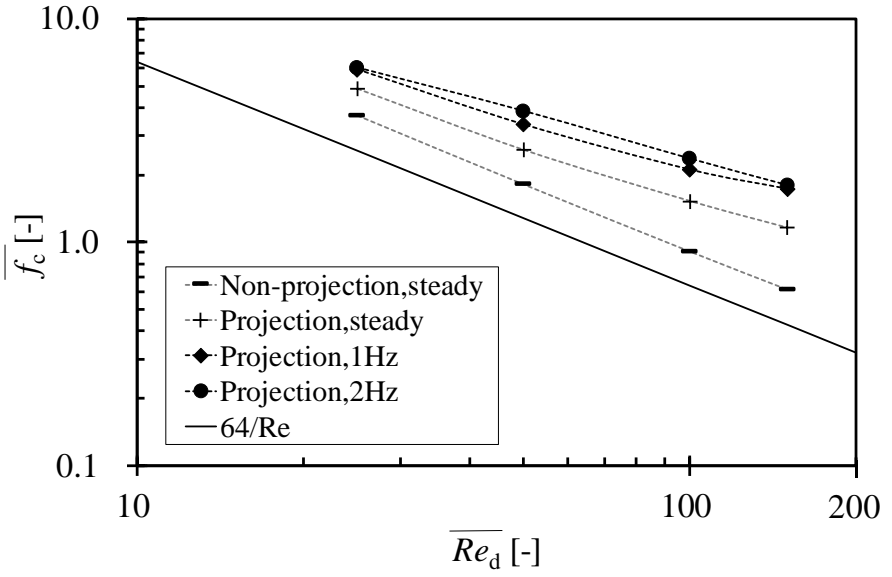

Fig. 10 Relationship between friction coefficient and time-averaged Reynolds number.

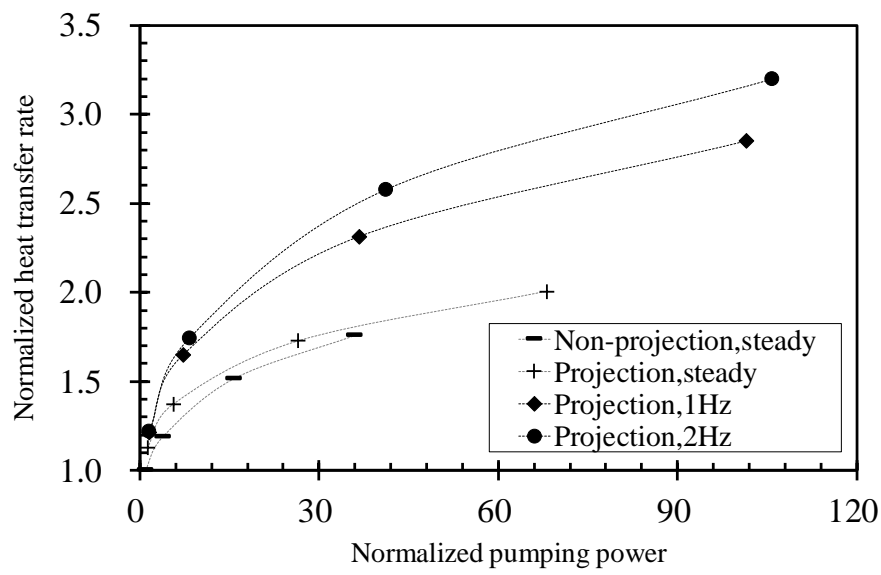

Fig. 11 Relationship between heat transfer rate and pumping power. Here, the results were normalized by those of Non-projection model in the case of $R e_{\mathrm{d}}=25$.

developed laminar flow in ducts $(=64 / R e)$. This is because the evaluation area is near the inlet boundary. Therefore, the flow is not fully-developed and friction drop becomes higher than the fully-developed flow.

On the other hand, Fig. 11 shows a relationship between heat transfer rate and pumping power. Here, the results were normalized by those of Non-projection model in the case of $R e_{\mathrm{d}}=25$. We can see that the increase of the heat transfer rate as compared to the pumping power in the case of the pulsating flow becomes extremely higher than that of the steady flow. This means that the heat transfer enhancement of the combination of the pulsating flow and the projection is fruitful from the viewpoint of the efficiency of the heat exchanger. Indeed, the application of the pulsating flow increases the pressure drop. However, much higher heat transfer performance can be obtained against the increase of the pumping power. Therefore, we can conclude that the application of the pulsating flow can enhance heat transfer while inhibiting an increase of a net time-averaged work of the fluid machineries.

\section{CONCLUSIONS}

We investigated the possibility of enhancing heat transfer in a narrow cooling channel that is generally observed in miniaturized heat exchangers by using pulsating flow. In particular, we evaluated the heat transfer performance of pulsating flow of Non-projection model and Projection model respectively while changing the time-averaged Reynolds number and the pulsating frequency through 3D-CFD analysis. The change of the flow pattern during pulsating flow generation was 
observed and the mechanism of heat transfer enhancement by pulsating flow was investigated. We obtained the following conclusions:

- The combination of the pulsating flow and the projection can enhance heat transfer significantly in a mm-scale narrow watercooling channel. In the range of our numerical conditions, the largest increase in heat transfer performance of $82 \%$ can be achieved. The mechanism of heat transfer enhancement by pulsating flow involves the generation of additional vortices around the projection by the sudden change of the flow pattern by stopping the flow intermittently.

- $\quad$ From the viewpoint of heat transfer efficiency, the application of the pulsating flow and the projection can enhance heat transfer performance while inhibiting an increase of a net timeaveraged work of the fluid machineries. This means that the total power consumption of heat exchange systems may be decreased by the application of the pulsating flow with felicitous manners.

- Through this paper, we clarified the qualitative effectiveness of the combination of the pulsating flow and the rib on the heat transfer enhancement. As our future research, in order to conduct quantitative evaluations, an increase a reliability of the CFD analysis will be discussed. In addition, we intend to carry out additional investigation of heat transfer efficiency of pulsating flow and the effect of pulsating flow on decreasing power consumption through actual experiment. In order to increase heat transfer performance while decreasing power consumption of the pumps, optimization of projection configuration and pulsating wave pattern should be investigated.

\section{ACKNOWLEDGEMENTS}

This work was supported by JSPS KAKENHI Grant Numbers JP16K18022, JP19K04249 and JP19K14916.

\section{NOMENCLATURE}

A

$d$

E

$f$

$h$

$l$

$\mathrm{Nu}$

$P$

$q$

$R e_{\mathrm{d}}$

$t$

$u$

$W$

$y$

Greek Symbols

$\alpha \quad$ thermal diffusivity $\left(\mathrm{m}^{2} / \mathrm{s}\right)$

$\Delta P \quad$ pressure difference $(\mathrm{Pa})$

$\theta \quad$ temperature (K)

$\lambda$ thermal conductivity $(\mathrm{W} /(\mathrm{m} \cdot \mathrm{K}))$

$v \quad$ kinematic viscosity $\left(\mathrm{m}^{2} / \mathrm{s}\right)$

$\rho \quad$ density $\left(\mathrm{kg} / \mathrm{m}^{3}\right)$

$\begin{array}{ll}\text { Subscripts } & \\ 1 & 1 \mathrm{~Hz} \\ 2 & 2 \mathrm{~Hz} \\ \mathrm{c} & \text { channel } \\ \mathrm{f} & \text { working fluid } \\ \text { in } & \text { inlet }\end{array}$

$\begin{array}{ll}\mathrm{m} & \text { area average } \\ \mathrm{max} & \text { maximum flow rate when pulsating } \\ \mathrm{p}, \text { pulsating } & \text { pulsating flow condition } \\ \mathrm{s} & \text { steady flow condition } \\ \mathrm{t} & \text { instantaneous value } \\ \mathrm{W} & \text { wall value } \\ x & \text { local value }\end{array}$

\section{REFERENCES}

Aliaga, D. A., Lamb, J. P. and Klein, D. E., 1994, "Convection heat transfer distributions over plates with square projections from infrared thermography measurements", International Journal of Heat and Mass Transfer, 37-3, 363-374. https://doi.org/10.1016/0017-9310(94)90071-X

Al-Turaihi, R. S., Fadhil, D. and Abed, A. M., 2019, "Effect of SemiCircle Rib on Heat Transfer Coefficient in a Rectangular Channel", Frontiers in Heat and Mass Transfer, 13-29. https://doi.org/10.5098/hmt.13.29

Bi, C., Tang, G. H. and Tao, W. Q., 2013, "Heat transfer enhancement in mini-channel heat sinks with dimples and cylindrical groove", Applied Thermal Engineering, 55, 121-132.

https://doi.org/10.1016/j.applthermaleng.2013.03.007

Colleoni, A., Toutant, A., Olalde, G. and Foucant, J. M., 2013, "Optimization of winglet vortex generators combined with riblets for wall/fluid heat exchange enhancement", Applied Thermal Engineering, 50, $1092-1100$.

https://doi.org/10.1016/j.applthermaleng.2012.08.036

Ditri, J., Hahn, J., Cadotte, R., McNulty, M. and Luppa, D., 2015, "Embedded Cooling of High Heat Flux Electronics utilizing Distributed Microfluidic Impingement Jets", InterPACK/ICNMM2015-48689, Proceedings of ASME 2015 International Technical Conference and Exhibition on Packaging and Integration of Electronic and Photonic Microsystems collocated with the ASME 2015 13th International Conference on Nanochannels, Microchannels, and Minichannels, San Francisco, CA.

Fukue, T., Hirose, K. and Yatsu, N., 2014, "Basic Study on Flow and Heat Transfer Performance of Pulsating Air Flow for Application to Electronics Cooling", Transactions of The Japan Institute of Electronics Packaging, 7-1, 123-131. https://doi.org/10.5104/jiepeng.7.123

Fukue, T., Ishizuka, M., Nakagawa, S., Hatakeyama, T. and Nakayama, W., 2010, "Resistance Network Analysis of Airflow and Heat Transfer in a Thin Electronic Equipment Enclosure with a Localized Finned Heat Sink", IHTC14-22979, Proceedings of the $14^{\text {th }}$ International Heat Transfer Conference, Washington D.C.

Fukue, T., Shirakawa, H., Hirose, K., Natsusaka, H. and Furusawa, T., 2016, "Possibility of Enhancement of Cooling Performance on Heating Surface by Using Intermittent Jet Flow", 2-02, Proceedings of IEEE CPMT Symposium Japan 2016, Kyoto, Japan.

Goshayeshi, H. R., Safaei, M. R., Goodarzi, M. and Dahari, M., 2016, "Particle size and type effects on heat transfer enhancement of Ferronanofluids in a pulsating heat pipe", Powder Technology, 301, 12181226.

https://doi.org/10.1016/j.powtec.2016.08.007

Hofmann, H. M., Kaiser, R., Kind, M. and Martin, H. 2007, "Calculations of Steady and Pulsating Impinging Jets - An Assessment of Widely used Turbulence Models", Numerical Heat Transfer, Part B, 51, 565-583.

https://doi.org/10.1080/10407790701227328 
Inukai, A., Takahashi, M., Hishida, M. and Tanaka, G., 2005, "Characteristic of Heat Transportation by an Oscillatory Flow (in Japanese)", Transactions of the Japan Society of Mechanical Engineers, Series $B$, 71-710, 131-138. https://doi.org/10.1299/kikaib.71.2515

Jaluria, Y., Sunder, A. and Benner, J. Z., 2020, "Thermal Management of Data Centers under Steady and Transient Conditions", Frontiers in Heat and Mass Transfer, 15-12.

https://doi.org/10.5098/hmt.15.12

Kasuga, Y., 2015, "Nonreflecting Boundary Condition of Waves (in Japanese)", On the Web, available from http://penguinitis.g1.xrea.com/ study/OpenFOAM/noreflect/noreflect.html (accessed May $3^{\text {rd }}, 2020$ ).

Kikuchi, Y., Ohno, Y., and Takahashi, M. 1995, "Combined Forced and Free Convective Heat Transfer from a Cylinder in Pulsating Cross-Flow (in Japanese)", Transactions of the Japan Society of Mechanical Engineers, Series B, 61-585, 202-207.

https://doi.org/10.1299/kikaib.61.1790

Liewkongsataporn, W., Patterson, T. and Ahrens, F., 2008, "Pulsating Jet Impingement Heat Transfer Enhancement”, Drying Technology, 26, 433442.

https://doi.org/10.1080/07373930801929268

Nozaki, F., 2019, "Non-Reflective Boundary Conditions in OpenFOAM", On the Web, available from https://caefn.com/openfoam/ bc-advective-wavetransmissive (accessed May $4^{\text {th }}, 2020$ ).

The Open CAE Society of Japan, 2016, Numerical Analysis of Heat Transfer and Fluid Flow by OpenFOAM (in Japanese), Morikita Publishing Co., Ltd., Japan, ISBN: 4627691017.

Panchal, S., Khasow, R., Dincer, I., Agelin-Chaab, M., Fraser, R. and Fowler, M., 2017, "Thermal design and simulation of mini-channel cold plate for water cooled large sized prismatic lithium-ion battery", Applied Thermal Engineering, 122, 80-90.

https://doi.org/10.1016/j.applthermaleng.2017.05.010
Reddy, S. R., Abdoli, A., Dulikravich, G. S., Pacheco, C. C., Vasquez, G., Jha, R., Colaco, M. J. and Orlande, H. R. B., 2015, "Multi-Objective Optimization of Micro Pin-Fin Arrays for Cooling of High Heat Flux Electronics with a Hot Spot", InterPACK/ICNMM2015-48242, Proceedings of ASME 2015 International Technical Conference and Exhibition on Packaging and Integration of Electronic and Photonic Microsystems collocated with the ASME 2015 13th International Conference on Nanochannels, Microchannels, and Minichannels, San Francisco, CA.

Saitoh, H. and Yoshioka, Y., 2010, "Effect of pulsating Amplitude on Flow Structure and Associated Heat Transfer around the Flat Plate Installed in Pulsating Duct Flow", Proceedings of the 21 st International Symposium on Transport Phenomena, Kaohsiung, Taiwan.

Shiibara, N., Nakamura, H. and Yamada, S., 2016, "Unsteady Characteristics of Turbulent Heat Transfer in a Circular Pipe at Sudden Acceleration and Deceleration of Flow", Proceedings of the $27^{\text {th }}$ International Symposium on Transport Phenomena, Honolulu, Hi.

Souma, A., Iwamoto, K. and Murata, A., 2010, "PIV Measurement of Pulsating Turbulent Pipe Flow for Energy Saving", Proceedings of the 21st International Symposium on Transport Phenomena. Kaohsiung, Taiwan.

Yang, F., Schultz, M., Parida, P., Colgan, E., Polastre, R., Dang, B., Tsang, C., Gaynes, M., Knickerbocker, J. and Chainer, T., 2015, "Local Measurements of Flow Boiling Heat Transfer on Hot Spots in 3D Compatible Radial Microchannels", InterPACK/ICNMM2015-48341, Proceedings of ASME 2015 International Technical Conference and Exhibition on Packaging and Integration of Electronic and Photonic Microsystems collocated with the ASME 2015 13th International Conference on Nanochannels, Microchannels, and Minichannels, San Francisco, CA.

Zhao, M. and Tian, Y., 2019, "Thermal Analysis of Heat Transfer Enhancement of Rib Heat Sink for CPU", Frontiers in Heat and Mass Transfer, 13-4. https://doi.org/10.5098/hmt.13.4 